\title{
Guided MAC to Enhance Quality of Experience of Video Data Transmission over Massive MIMO
}

\author{
Diwakar Bhardwaj \\ Department of Computer Engineering \& Applications, GLA University, Mathura \\ Corresponding Author : diwakar.bhardwaj@gla.ac.in
}

\begin{abstract}
Massive MIMO (M-MIMO) system comprises of multiple number of antennas to achieve energy- efficiency and large gains in spectral-efficiency in comparison to existing MIMO technology. High speed and Quality of Experience (QoE) of video data over wireless communication has always been a challenge for the researchers due to scarcity of the bandwidth, fading and interference. The channels with high noise corrupt the transmitted video and results in poor QoE of at the receiver. Therefore, to maintain the quality of transmitted video, it is highly desirable to identify noisy channels and avoid transmission over them. This paper deals with QoE of the transmitted video over Massive MIMO channels. The channels are categorized into two categories: good and bad depending upon the value of Signal to Interference and Noise Ratio (SINR). A channel above the minimum acceptable value (threshold) of SINR is categorized as good channel otherwise bad channel. A Guided MAC layer (GMAC) protocol is designed to transmit the video data over good channels only and to discard the transmission over bad channels.
\end{abstract}

Keywords: Massive MIMO; QoE; Channel state information; SINR; MAC layer; Single description coding (SDC).

\section{INTRODUCTION}

With improved wireless technologies in society the demand of video transmission is increased. Therefore, the wireless internet devices face the demand of proliferating high data rate services. The experts of wireless industries have reached the conclusion that incremental technologies $(1 \mathrm{G}$ to $4 \mathrm{G})$ to be unsuccessful to meet the prospective future data demands.

A 4G system must carry abilities defined by ITU in Advanced. Various challenges addressed in 4G are: 1) High data rate, 2) High Mobility, 3) High capacity, 4) Lower E2E delay, 5) and massive device communication. The current existing technology i.e., 4G uses Conventional MIMO which uses at most 4 antennas for transmission of data. By which it fails to transmit a high quality data (HD+ video) with low latency. Other challenges include the need of higher data rate, low cost, low end to end latency, enormous device communication with better QoS. To overcome these challenges (Ping Wang et al., 2015) suggested the use of 5G technologies which allow the world with huge capacity, massive connectivity and future internet.

Evolution of new wireless technologies in the past and current decade gave significant growth of different wireless networks 5G technologies, like Massive Multiple Input Multiple Output (M- MIMO), millimeter waves (mm Waves), Machine to Machine communication (M2M communication) etc., which have facilitated fast data transmission between the communicating devices with certain challenges like interference and pilot contamination. M-MIMO can be a promising technology of the future having large potential of high data speed, energy efficiency and spectral 
efficiency through the use of a large amount of antennas at base station to serve the users.

While promising technology, transmission of video data over M-MIMO still presents various research challenges like poor QoE. We have proposed a method to reduce the co-channel interference and augment the quality of the transmitted video. In this paper, we have used Channel State Information (CSI) to observe the status of various channels and categorize them as good or bad. Transmissions over good channels reduce packet error and retransmissions of the corrupted packets which result in effective bandwidth availability. The guided MAC mechanism of the proposed method restricts the admission of the packets over bad channels.

\section{LITERATURE SURVEY}

The review has revealed the challenges in low- latency transmission and improving QoE of video data over MMIMO. (Ping Wang et al., 2015) have proposed a QoS aware cell association mechanism using Massive MIMO. A user selects a cell in HetNet, based on the data rate broadcasted by the all available cell and then calculate average data rate, If the required data rate is less than the calculated one, user switches to other cell. (Alexei Ashikhmin1 et al., 2014) proposed an interference reduction in multi cell MIMIO system using outer multi-cellular pre coding. (Emil Björnso and Marios Kountouris, 2013) have proposed an energy efficient approach using Large Scale Fading Pre coding for combining messages obtained from different cells. The authors proposed a dispersed topology that allows for high spatial reuse while reducing energy consumption without compromising QoS. (Dasari Srinivasa Rao and Victor Dhason Berlin Hency, 2017), focused on Joint User Selection and Scheduling (JUSS) rather than collecting Channel State Information (CSI) from all users. Interference issues in Random Access Network are addressed by (E. Pateromichelakis et al., 2017) through interference management schemes. This research work elaborates three interference management drivers, for service- tailored optimization to improving cell-edge throughput, energyefficiency and reducing the signaling overhead using Base Station (BS) clustering and context-awareness.

(Lu Lu et al., 2014) explore the effect of pilot detoxification in massive MIMO. Within the same cell and neighboring cells, the massive MIMO pilot sequence must be orthogonal. However, the number of orthogonal Pilot sequences available within a given bandwidth is restricted. (Jia Liu et al., 2017) discovered the tradeoffs between throughput, delay, and the inaccuracy of the attainment of the CSI in M-MIMO cellular network systems. To demonstrate the transitioning phenomenon in the steady-state queue-length deviation in CSI, the authors characterized the queue-lengths scaling performance and the congestion control rate scaling performance. (Erik G. Larsson et al., 2014) suggested Massive MIMO as a golden mine for researchers because of challenges like channel reciprocity, pilot contamination. (A. K Mishra and S Gaur, 2016) analyzed the use of information theory to demonstrate the ostensible benefits of massive MIMO, as well as implementation problems such as channel estimation, identification, and precoding schemes. (Deli Qiao et al., 2014) proposed an architecture and compared the performance of co-located and distributed arrangement of antennas for Area Spectrum Efficiency (ASE) and Area Energy Efficiency. In (Haifan Yin, 2012), multi-cell interference-limited cellular networks, the issue of channel estimation was discussed. During the channel estimation method, the authors developed a low-rate coordination among cells. The channels' second-order statistical information is used in the coordination process. (H Q Ngo, Hien Quoc, and Erik G. Larsson, 2012) proposed an approach which exploits the asymptotic orthogonality of the channel vectors in MMIMO. Authors make use of the covariance matrix of the received signals to estimate the user's channel. To make this estimation more effective authors also consider iterative least- square with projection (ILSP) algorithm. In a single-cell scenario where propagation is dominated by independent Rayleigh fading, (Yang, Hong, and Thomas, 2013) compared linear pre-coders, conjugate beam formation, and zero-forcing, (Kim et al., 2017) summarized various SDC based video data transmission over MIMO systems with cross layer architecture and investigated the UEP solutions for video transmission over Massive MIMO. Using the classic convex optimization approach, (Xiang Chen et al.) proposed an energy efficient mechanism to increase the Quality of Experience of SDC dependent video transmission over MIMO. 


\section{METHODOLOGY}

We assume a two-dimensional cellular network composed of ' $k$ ' hexagonal cells with one base station and ' $n$ ' users in each cell. There are ' $\mathrm{M}$ ' antennas at the base station and one antenna at the user equipment (UE).

This section proposes a mathematical model for the hypothesis of classifying the channels in good or bad category and observing the QoE at the receiver. Estimating expected PSNR at receiver before transmission over a specific channel offers a better solution to decide whether to transmit over that channel or not. The proposed protocol considers the small/large scale fading channel model to transmit a video over it. The results obtained in this work outperform from the existing Massive MIMO technology [9-19].

\section{SYSTEM MODEL}

L-cell SU-MIMO systems is considered with ' $\mathrm{K}$ ' single-antenna users and one BS with ' $\mathrm{N}$ ' antennas in each cell. The channel coefficient is denoted by h.i,k,l,n, and is modeled as

$$
h=g * d
$$

Where $\mathrm{g}$, , , and $\mathrm{d}$, , denote complex small scale and large scale fading coefficients, respectively. The channel matrix for all $\mathrm{K}$ users in 1-th cell to i-th BS can be given as

$$
H=G * D
$$

where $\mathrm{G}$, and $\mathrm{D}$, are complex small scale and large scale fading. The channel matrix $(\mathrm{H})$ is given as:

$$
\left[\begin{array}{ccr}
h_{11} & h_{12} & h_{13} \ldots \ldots . h_{1 \times n T} \\
h_{21} & h_{22} & h_{23} \ldots \ldots . h_{2 \times n T} \\
h_{31} & h_{32} & h_{33} \ldots \ldots . h_{3 \times n T} \\
h_{n R \times 1} & h_{n R \times 2} & h_{n R \times 3} \ldots \ldots . . h_{n R \times n T}
\end{array}\right]
$$

Here $h_{j}$ represents the channel between $i^{\text {th }}$ and $j^{\text {th }}$ antennas of transmitter and receiver, respectively.

\section{CALCULATION OF GOOD AND BAD CHANNELS}

Let $\mathrm{X}(\mathrm{t})$ be the transmitted signal from a transmitter equipped with an array of $\mathrm{N}_{\mathrm{T}}$ transmitting antennas then the received signal $\mathrm{Y}(\mathrm{t})$ at a receiver with an array of $\mathrm{N}_{\mathrm{R}}$ receiving antennas can be given as follows:

$$
Y(t)=H * X(t)+n(t)
$$


Here $n(t)$ is a zero mean noise vector with complex Gaussian distribution and calculated as follows:

$$
n(t)=\text { mean }+s d * \operatorname{rand} n
$$

Based on the channel strength (CSI), channel matrix (H) is categorized into good \& bad channels. If signal strength received at the receiver $(\mathrm{Y}(\mathrm{t}))$ is greater than or equal to a $\mathrm{QoE}$ threshold value $\left(\mathrm{QoE}_{\mathrm{Th}}=25 \mathrm{~dB}\right.$, acceptable signal strength as per ITU), for a given input signal $\mathrm{X}(\mathrm{t})$, channels are categorized as good otherwise bad:

$$
\begin{gathered}
H_{\text {good }}=\left[\begin{array}{lll}
g_{11} & g_{12} & g_{13 \ldots \ldots} \ldots \\
g_{21} & g_{22} & g_{23} \ldots . \\
g_{31} & g_{32} & g_{33 \ldots \ldots} \ldots . . \\
g_{41} & g_{42} & g_{43} \ldots
\end{array}\right] p \times s \\
H_{\text {bad }}=\left[\begin{array}{lll}
b_{11} & b_{12} & b_{13 \ldots \ldots} \ldots \ldots \\
b_{21} & b_{22} & b_{23} \ldots . \\
b_{31} & b_{32} & b_{33} \ldots \ldots \\
b_{41} & b_{42} & b_{43} \ldots
\end{array}\right] u \times v
\end{gathered}
$$

Here $H_{\text {good }}$ and $H_{b a d}$ are the matrices representing a set of good and bad channels, respectively, depending upon the received signal value at the receiver.

\section{Algorithm for GMAC}

1. Let $T$ be the transmitter and $R$ be the receiver with $M$ and $N$ antennas, respectively.

2. $C S /$ th $=25 d B . / / C S I$ Threshold

3. $H M N=G M N{ }^{*} D / / /$ CSI Estimation

4. for $i=1$ to NT do //nos. of antennas at transmitter

5. for $j=1$ to NR do // nos. of antennas at receiver

6. $Y i j=H i j * X i j+n(t) / /$ Signal Estimation at sender

7. If Yij $>=C S /$ Th then //Channel Categorization

8. $\operatorname{HGood}(, j)=H(i, j)$

9. $\quad$ Else $\operatorname{HBad}(, j)=H(i, j)$.

10. End if

11. End for 


\section{End for}

13. // Channel Accessing by GMAC for data transmission over Good Channel

14. for $i=1$ to $\mathrm{Np}$ do

15. for $j=1$ to $\mathrm{Ns}$ do

16. $\operatorname{Yij}(t)=\operatorname{HGood}(i, j) \times X i j+n(t)$

17. If $\operatorname{Yij}(t)>25 d B$ then

18. Channel is available for Video transmission

19. Else

20. Channel is avoided for Video transmission

21. Yij(t) is recalculated after $\delta t$ time

22. Endf

23. End for

24. End for

25. End.

The proposed method forces MAC layer to access the good channels and transmit the video data over these channels only and avoid transmission over bad channels to achieve required acceptable QoE. Therefore, to obtain acceptable good quality at the receiver eq. (4) is modified as follows:

$$
\begin{array}{r}
\mathrm{Y}(\mathrm{t})=\mathrm{Hgood}_{\text {goo }} * \mathrm{X}(\mathrm{t})+\mathrm{n}(\mathrm{t})(8) \text { Where } H_{\text {good }} \geq \text { CSITh } \\
Y(t)=H_{\text {good }} * X(t)+n(t)
\end{array}
$$

Where $H_{\text {good }} \geq C S I_{T h}$

\section{CHANNEL ACCESSING SCHEME OF MAC LAYER}

Medium Access (MAC) layer of the proposed approach is designed to checks whether the available channels are good or bad channels depending upon the CSI threshold value, and make available the good channels only to the station which wins the contention. The stations who do not get channels access take a random back-off. Bad channels are discarded by the MAC layer for the current transmission and reestablishes the good and bad channels for a new flow. 


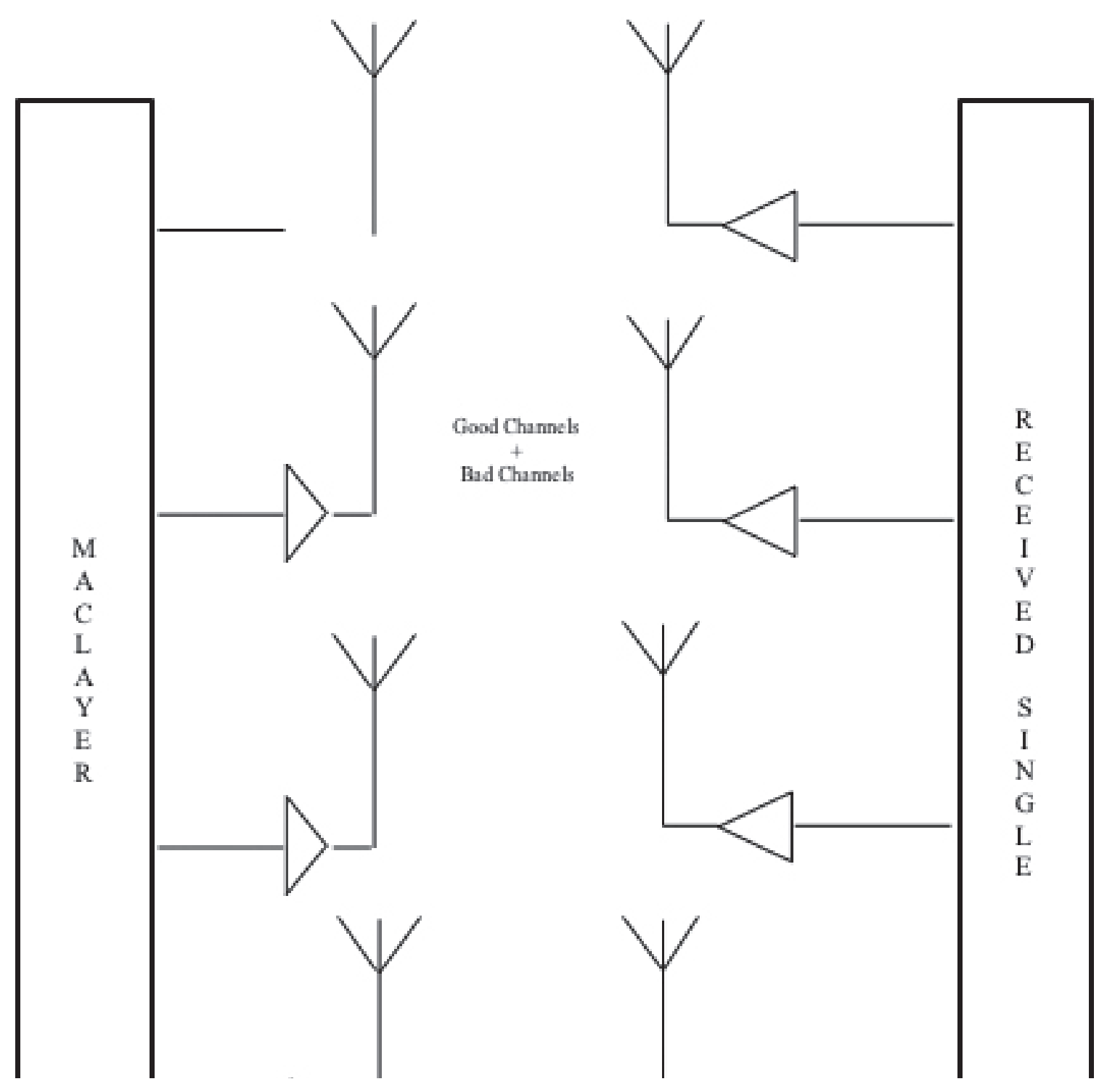

Figure 1. Classification of channels as Good and Bad

\section{RESULTS AND DISCUSSION}

This section discusses about results analysis and validation carried out for the proposal. NS-2 is used to simulate the environment. A network of ' 50 ' stations connected via base stations having a matrix of $64 \times 64$ antennas. A $40 \mathrm{~dB}$ video is transmitted over the noisy channels having noise ranging from $15 \mathrm{~dB}$ to $50 \mathrm{~dB}$. The PSNR values obtained at receiver using proposed protocol and limited CSI information are compared in Fig. 2. A video packet received at receiver having PSNR greater than $25 \mathrm{~dB}$ is acceptable and found with satisfactory QoE. 


\section{(i) All Channels are Noiseless}

The performance of both the methods, Limited CSI and GMAC is observed same when channels are noiseless (Fig. 2). The quality of the video packets transmitted over noiseless channels and received at receiver is obtained greater than $25 \mathrm{~dB}$ in both the methods. GMAC behaves similar to Limited CSI method and transmit data over all channels without excluding any channel.

\section{Limited CSI Vs GMAC}

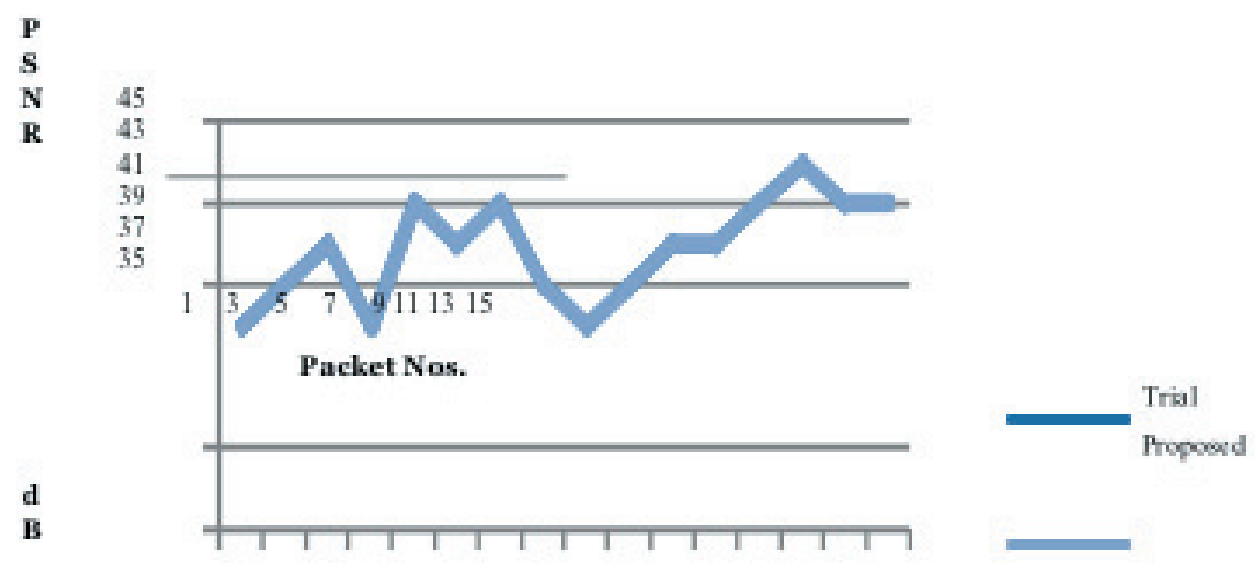

Figure 2. PSNR value received at receiver in noiseless channels

\section{(i) Channels are Noisy}

In the simulated environment channels, some of the channels are assumed noisy for time span 500ms with signal strength below $25 \mathrm{~dB}$. PSNR values of the transmitted video packets are compared at receiver with the PSNR values at sender. From Fig. 3, it can be observed that PSNR value of the received packet at receiver is obtained better in the proposed GMAC method because it exclude the noisy channels from the transmission process and forward the packets to noiseless channels (if any) for transmission.

Packets are transmitted over all 64 channels in limited CSI information method; therefore, the PSNR obtained of the packets which are passed through the noisy channels is low and hence contribute to bad QoE. It can be observed from the Fig. 4 that some of the packets (packet nos. 21 to 25, 50, 51 and 60) are poor QoE (below $25 \mathrm{~dB}$ ) at receiver. Therefore, The QoE in Limited CSI method is observed poor as compared to proposed GMAC method. On the other hand, GMAC keep on calculating CSI of the channels after every 500ms and stop transmission over a channel if its CSI goes below $25 \mathrm{~dB}$. From the Fig. 3, we can observe that all packets are having satisfactory or good QoE (PSNR $>25 \mathrm{~dB}$ ). 


\section{Limited CSI Info vs GMAC}

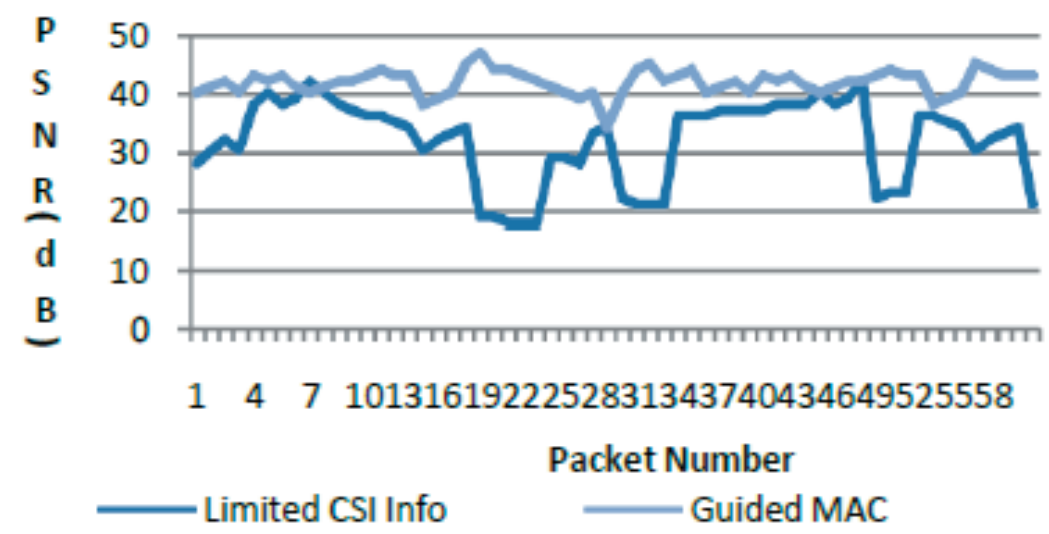

Figure 3. PSNR Value of the received packet at Receiver in noisy channels

Fig. 4(a) is an actual video frame which is transmitted using both the methods; the frame Fig. 4(b) and Fig. 4(c) are received at receiver using Limited CSI method and GMAC respectively. From the Fig. 4(b) and Fig. 4(c), it can be observed that frame QoE in GMAC is better than Limited CSI.

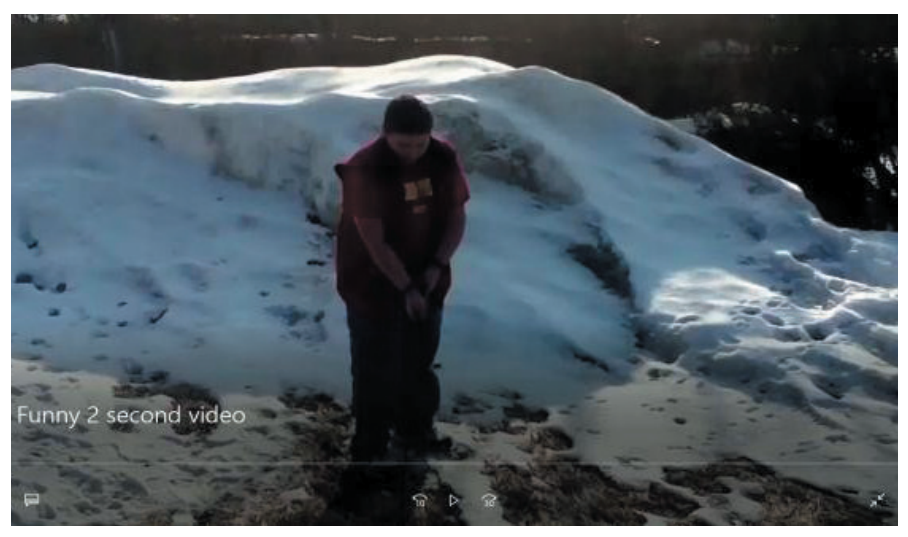

Figure 4(a): Actua 1 video frame.

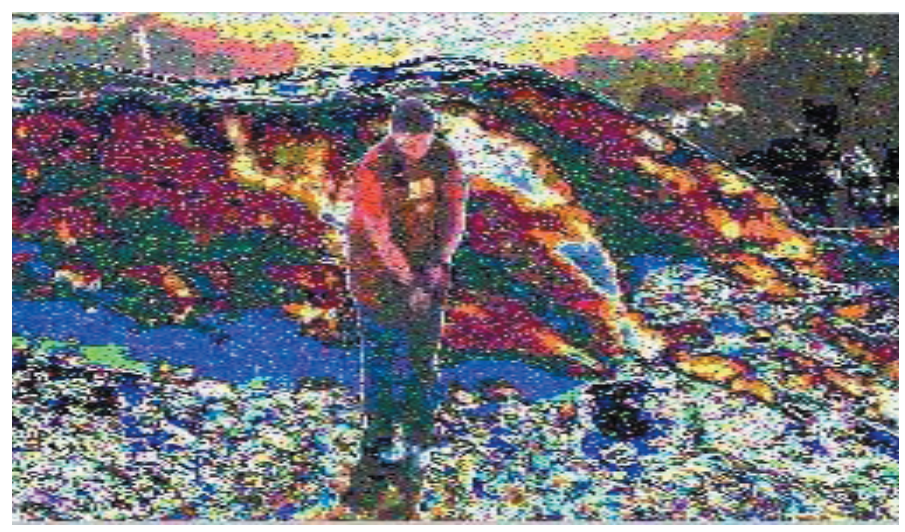

Figure 4(b): A Video frame received at receiver in Limited CSI Method. 


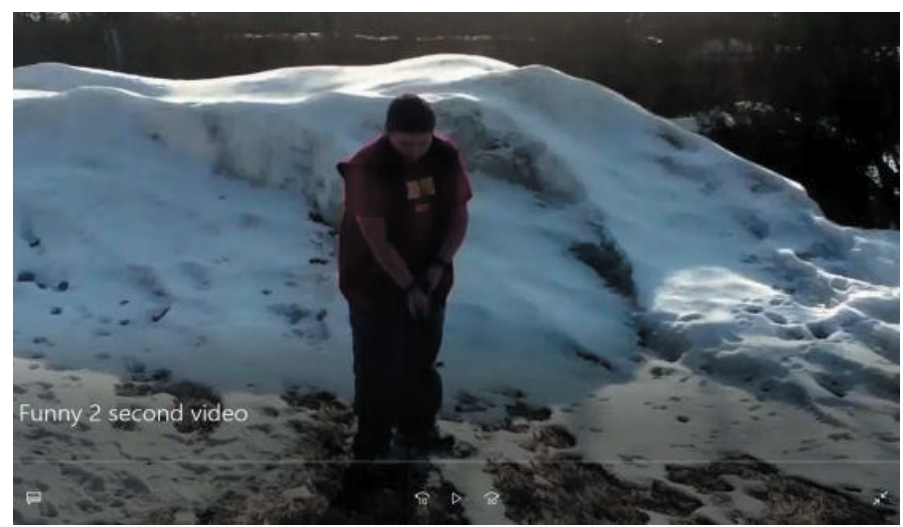

Figure 4(c): A video frame in GMAC Method

End to End delay in GMAC is $30 \%$ higher than Limited CSI method as number of available channels for transmission is less and more packets are sent on single channel. But this high end to end delay (E2E) (maximum up to $10 \mathrm{~ms}$, Fig. 5) is observed within the limit and does not make any effect on the QoE.

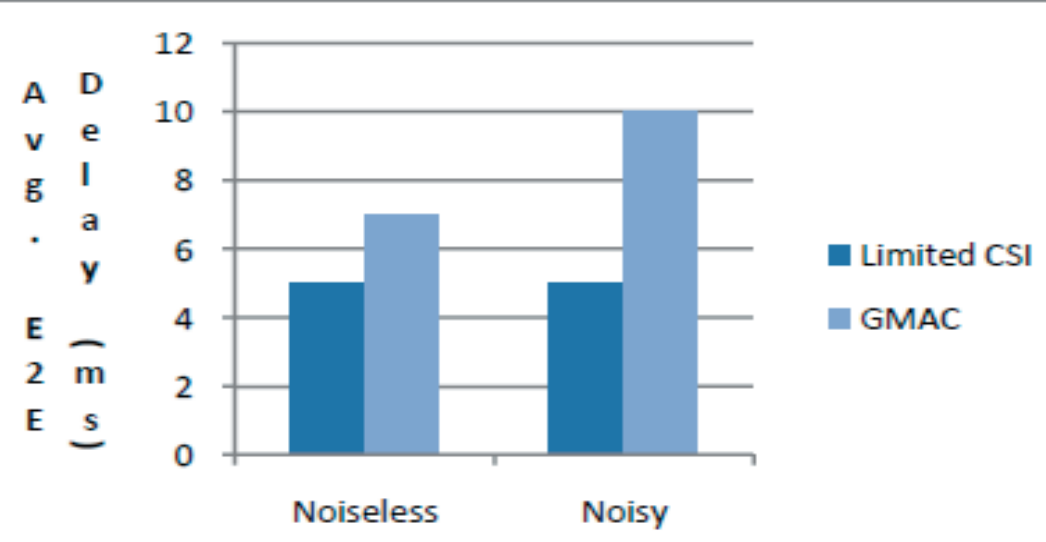

Figure 5: End to End Delay in Limited CSI and GMAC

\section{CONCLUSION}

Proposed GMAC method for channel accessing addresses the challenges of QoE for video transmission over Massive MIMO. GMAC outperforms the existing Limited CSI method with high (30\%) end to end delay. GMAC supports the importance of channel state information to transmit the data. GMAC discard the packet transmission and do not work when all the channels are noisy $(<25 \mathrm{~dB})$. We leave this to the future researchers to contribute and find the solution.

\section{REFERENCES}

Ping Wang, Wei Song, Dusit Niyato, and Yong Xiao. (2015). QoS-Aware Cell Association in 5G Heterogeneous Networks with Massive MIMO. IEEE Network, 76-82. 
Ashikhmin, A., Marzetta, T. L., \& Li, L. (2014). Interference reduction in multi-cell massive MIMO systems I: Large-scale fading precoding and decoding. arXiv preprint arXiv:1411.4182

Björnson, E., Kountouris, M., \& Debbah, M. (2013). Massive MIMO and small cells: Improving energy efficiency by optimal soft-cell coordination. In ICT 2013 (pp. 1-5). IEEE.

Rao, D. S., \& Hency, V. D. B. (2017). QoS-based Joint User Selection and Scheduling for MU-MIMO WLANs. Journal of Telecommunications and Information Technology, (4), 17-24.

Pateromichelakis, E., Celik, H., Fantini, R., Bulakci, Ö., Campoy, L. M., Gutierrez-Estevez, D. M., ... \& Yang, Y. (2016). Interference management enablers for $5 \mathrm{~g}$ radio access networks. In 2016 IEEE Conference on Standards for Communications and Networking (CSCN) (pp. 1-7). IEEE.

Lu, L., Li, G. Y., Swindlehurst, A. L., Ashikhmin, A., \& Zhang, R. (2014). An overview of massive MIMO: Benefits and challenges. IEEE journal of selected topics in signal processing, 8(5), 742-758.

Liu, J., Eryilmaz, A., Shroff, N. B., \& Bentley, E. S. (2017). Understanding the impacts of limited channel state information on massive MIMO cellular network optimization. IEEE Journal on Selected Areas in Communications, 35(8), 1715-1727.

Erik G. Larsson,Ove Edfors, Fredrik Tufvesson Thomas L. Marzetta - (2014). Massive MIMO for next generation wireless systems. IEEE Communications Magazine 52.2, 186-195.

Mishra A. K., Gaur S. (2016). Review of The Pilot Contamination Problem For Massive MIMO And Possible Solution. International Journal of Engineering Sciences and Research Technology 5 (7), 538-541.

Qiao, D., Wu, Y., \& Chen, Y. (2014). Massive MIMO architecture for 5G networks: Co-located, or distributed?. In 2014 11th International Symposium on Wireless Communications Systems (ISWCS) (pp. 192-197). IEEE.

Yin, H., Gesbert, D., Filippou, M., \& Liu, Y. (2013). A coordinated approach to channel estimation in large-scale multiple-antenna systems. IEEE Journal on selected areas in communications, 31(2), 264-273.

Ngo, H. Q., \& Larsson, E. G. (2012). EVD-based channel estimation in multicell multiuser MIMO systems with very large antenna arrays. In 2012 IEEE International Conference on Acoustics, Speech and Signal Processing (ICASSP) (pp. 3249-3252). IEEE.

Yang, H., \& Marzetta, T. L. (2013). Performance of conjugate and zero-forcing beamforming in large-scale antenna systems. IEEE Journal on Selected Areas in Communications, 31(2), 172-179.

Kim, S. J., Suk, G. Y., Lee, J. S., \& Chae, C. B. (2017). QoE-aware scalable video transmission in MIMO systems. IEEE Communications Magazine, 55(8), 196-203.

Chen, X., Hwang, J. N., Wang, C. Y., \& Lee, C. N. (2014). A near optimal QoE-driven power allocation scheme for SVC-based video transmissions over MIMO systems. In 2014 IEEE International Conference on Communications (ICC) (pp. 1675-1680). IEEE. 